\title{
Cáncer renal incidental en pacientes de edad geriátrica: hallazgos clínicos e histopatológicos
}

\author{
L. Gómez Pérez, A. Budía Alba, F.J. Delgado Oliva, J.L. Ruiz Cerdá, M.A. Bonillo García, \\ J.F. Jiménez Cruz
}

Servicio de Urología. Hospital Universitario La Fe. Valencia.

Actas Urol Esp 2006; 30 (2): 139-144

\begin{abstract}
RESUMEN
CÁNCER RENAL INCIDENTAL EN PACIENTES DE EDAD GERIÁTRICA: HALLAZGOS CLÍNICOS E HISTOPATOLÓGICOS

Objetivo: evaluar las características clínicas y patológicas del CCR en nuestra serie de tumores, analizando su impacto en el grupo de edad de mayores de 65 años.

Material y método: Se estudiaron 300 pacientes con adenocarcinoma renal (CCR). En 252 se realizó un estudio del ADN tumoral mediante citometría de flujo e histopatológico de las piezas quirúrgicas. Según los criterios de la Sociedad Española de Geriatría, los pacientes fueron divididos en dos grupos en función de la edad, mayores y menores de 65 años. Las características clínicas e histopatológicas fueron comparadas entre ambos grupos. También encontramos diferencias significativas al comparar la supervivencia actuarial cáncer específica de modo global, con mayor supervivencia de los pacientes menores de 65 años

Resultados: De los 300 pacientes, 103 (33,3\%) se incluyeron en el grupo de edad geriátrica. No hallamos diferencias significativas entre los grupos al comparar estadio, tamaño tumoral o tratamiento realizado, aunque encontramos un mayor índice de recidivas en los pacientes de edad geriátrica. El análisis del DNA evidenció un mayor porcentaje de aneuplodías en el grupo en edad geriátrica. La supervivencia actuarial cáncer específica fue superior en el grupo de menos de 65 años.

Conclusiones: El CCR en la edad geriátrica, tiene unas características clínicas y patológicas similares al resto de pacientes en nuestra serie. Sin embargo existen diferencias en la supervivencia media y actuarial, que es menor en el paciente de más de 65 años por el mayor porcentaje de aneuploidías detectado y la mayor tasa de recidivas en tumores organoconfinados en este grupo de edad.
\end{abstract}

Palabras clave: Cáncer renal. Geriatría. Análisis DNA.

\section{ABSTRACT}

RENAL CANCER IN ELDERLY: CLINICAL AND HISTOPATHOLOGICAL FINDINGS

Objetive: to evaluate the clinical and pathological characteristics of the CCR in our series of tumors, analyzing its impact in the group of age greater than 65 years.

Material and methods: 300 patients with renal adenocarcinoma (CCR) were studied. In 252, ploidy pattern of DNA and pathologic characteristics of the surgical pieces were done. According to the criteria of the Spanish Society of Geriatrics, the patients were divided in two groups based on the age, greater and smaller of 65 years. The clinical and pathologic characteristics were compared between both groups.

Results: 103 of the 300 patients (33.3\%) were included in the geriatric group. We did not found differences between both groups comparing stage, tumoral volume or treatment realized, but found differences in DNA ploidy pattern, recurrences and survey.

Conclusions: The RCC in the elderly has a few clinical and pathological characteristics similar to the rest of patients in our series. Nevertheless differences exist in the average and actuarial survival, which is minor in the patient of more than 65 years, cause the percentage of detected aneuploidies and number of recurrences in not confined tumors in this group of age.

Keywords: Renal cancer. Elderly. DNA ploidy. 
$\mathrm{E}^{1}$ adenocarcinoma renal (CR) presenta una incidencia aproximada del 5\% y ocasiona el $1-2 \%$ de todas las muertes por cáncer ${ }^{1}$. Dos tercios de estos tumores están localizados al diagnóstico y son potencialmente curables mediante tratamiento quirúrgico. Sin embargo el cáncer renal es el más letal de los cánceres urológicos, con una tasa de mortalidad del $40 \%$ comparado con la tasa del $20 \%$ asociada a otros tumores urológicos incluyendo el de próstata o vejiga ${ }^{2}$. A pesar del tratamiento radical, el $40 \%$ desarrollarán metástasis y fallecerán ${ }^{3}$.

Los factores pronósticos que han demostrado su valor independiente de supervivencia y progresión en la mayoría de los estudios publicados en la literatura son el grado nuclear, el estadio al diagnóstico en la actual clasificación TNM $2002^{4,5}$, y la ploidía del ADN tumoral, aunque éste último todavía con un papel controvertido ${ }^{6}$. El tipo histológico también ha mostrado su valor pronóstico, tras su reciente reclasificación ${ }^{3}$.

Sin embargo, la aplicación clínica de estos factores no consigue identificar con completa exactitud aquellos tumores que progresarán. Así, el $10-20 \%$ de los tumores órgano-confinados, a priori de buen pronóstico, desarrollarán metástasis y fallecerán por dicha causa. Por ello, son necesarios más estudios que aporten más información y permitan identificar con mayor exactitud ese grupo de pacientes candidatos a un seguimiento más estricto o a terapias adyuvantes $^{7,8}$. Recientemente, en un intento de mejorar la predicción pronóstica han surgido nuevos sistemas integrados de estadificación, combinando el TNM con otras variables clínicas y patológicas, que permiten estratificar en categorías pronósticas a los pacientes, calculando mediante herramientas estadísticas la probabilidad de supervivencia para cada paciente ${ }^{9}$.

Otro factor analizado en la literatura ha sido la influencia de la edad en el $\mathrm{CR}$, así recientes trabajos han evaluado la importancia de la edad en el $\mathrm{CR}$ e incluso han caracterizado los tipos histológicos más frecuentes ${ }^{10}$, riesgo de recurrencia $^{3}$ e implicación pronóstica $^{11}$ en función del grupo de edad al que pertenecen (jóve-

Tabla 1

$\mathrm{p}=0,887$ nes vs. ancianos). Sin embargo los resultados publicados son controvertidos.

El objetivo de este estudio ha sido evaluar la influencia de la edad en las características clínicas y patológicas del $\mathrm{CR}$ en nuestra serie de tumores, analizando y comparando las características clínicas y tumorales del grupo de edad mayor o igual de 65 años con los menores de esta edad.

\section{MATERIAL Y MÉTODO}

Hemos estudiado de forma retrospectiva a 300 pacientes con diagnóstico de CR tratados quirúrgicamente en nuestro Servicio entre los años 1992 a 2001. En 252 de los pacientes incluidos, se realizó un estudio del contenido de ADN tumoral mediante citometría de flujo. Todos los pacientes incluidos tenían estudio histopatológico de las piezas quirúrgicas. Para dicho estudio, los tumores fueron reclasificados según la actual $6^{\text {a }}$ edición de la clasificación TNM 2002 (Tabla 1). La mediana de seguimiento fue de 37,03 meses.

En este trabajo, para analizar la influencia de la edad en el CR, los pacientes fueron divididos en dos grupos: mayores o iguales a 65 años (grupo I) y menores de 65 años (grupo II), según los criterios de la Sociedad Española de Geriatría, que establece la edad geriátrica en un rango de 65-75 años. Las características clínicas y tumorales fueron comparadas entre ambos grupos.

$\mathrm{El}$ análisis molecular del $\mathrm{ADN}$ se realizó sobre células obtenidas de muestras en fresco de la pieza quirúrgica en los pacientes más recientes (1996-2001) y sobre muestras desparafinadas en los pacientes intervenidos antes de 1996. Tras un proceso de disgregación enzimática con pepsina se añade un reactivo comercial Coulter DNA-Prep Reagents Kit ${ }^{\circledR}$ (Coulter Corporation, Miami, Florida, USA) y se procede a la lectura por el citómetro de flujo. Como muestra control se utilizó

Distribución de pacientes según estadio y grupo de edad

\begin{tabular}{lcccc}
\hline & $\begin{array}{c}\text { Estadio I } \\
\mathbf{n ~ ( \% )}\end{array}$ & $\begin{array}{c}\text { Estadio II } \\
\mathbf{n ~ ( \% )}\end{array}$ & $\begin{array}{c}\text { Estadio III } \\
\mathbf{n ~ ( \% ) ~}\end{array}$ & $\begin{array}{c}\text { Estadio IV } \\
\mathbf{n ~ ( \% )}\end{array}$ \\
\hline Edad < 65 años & $54(27,8)$ & $40(20,6)$ & $69(35,6)$ & $31(16)$ \\
Edad > 65 años & $29(92,3)$ & $18(18,2)$ & $38(38,4)$ & $14(14,1)$ \\
Valor p & 0,78 & 0,62 & 0,63 & 0,66 \\
\hline
\end{tabular}


sangre periférica de enfermos quirúrgicos sin enfermedad neoplásica y con hemograma normal. Las muestras tumorales fueron consideradas diploides cuando el índice de DNA estaba entre 0,9 y 1,1 (cociente entre el canal del pico $\mathrm{G}_{0} / \mathrm{G}_{1}$ de la muestra y el canal del pico $\mathrm{G}_{0} / \mathrm{G}_{1}$ de las células control diploides normales). Además las muestras fueron consideradas diploides cuando sólo un pico era detectado en el análisis y aneuploides cuando se hallaban 2 picos. La mezcla de una muestra control con la del paciente nos permitía confirmar si se trataba de una hipo o hiperploidía.

La progresión clínica fue definida como recidiva loco-regional, a distancia o en riñón contralateral.

$\mathrm{El}$ análisis estadístico se realizó con programa estadístico SPSS versión 12.0 (SPSS, Inc., Chicago, Illinois). El valor de p considerado significativo fue menor de 0,05. Las variables a estudio fueron categorizadas siguiendo criterios clínicos o de tamaño muestral. Para las comparaciones de las características clínicas e histopatológicas entre los grupos de edad definidos, se utilizaron las pruebas de Chi cuadrado y el test de Fischer cuando algunas de las frecuencias esperadas era menor de 5 . La supervivencia actuarial cáncer específica fue calculada con las curvas de Kaplan Mayer y el test de log- rank para la comparación de grupos. Para el análisis multivariante se utilizó el modelo proporcional de regresión de Cox, como modelo más adecuado para determinar la influencia de unas variables explicativas cuantitativas o categóricas sobre una variable respuesta (supervivencia).

\section{RESULTADOS}

De los 300 pacientes, 197 $(65,7 \%)$ tenían menos de 65 años (26 a 64 años, con mediana de edad 54 años) y $103(33,3 \%)$ se incluyeron en el grupo de pacientes en edad geriátrica (desde 65 a 83 años, con mediana de edad 70,2 años) en el momento del diagnóstico.

Cuando comparamos la muestra según el estadio patológico categorizado en órgano-confina-

Tabla 2

$\mathrm{P}=0,169$ dos y no órgano-confinados, observamos que en el grupo I los tumores estaban órgano-confinados al diagnóstico en 55 pacientes $(52,4 \%)$, frente a los 102 pacientes $(51,7 \%)$ del grupo II $(\mathrm{p}=0,813)$. El estadio patológico más frecuente fue el III en ambos grupos (Tabla 1). No encontramos diferencias en el número de pacientes con adenopatías positivas al diagnóstico (20\% en grupo I vs. $21,6 \%$ en grupo II, $\mathrm{p}=0,428$ ), ni con metástasis (8\% en grupo I vs. $5,7 \%$ en grupo II, $\mathrm{p}=0,441)$.

Respecto a las características histopatológicas del tumor, el grado nuclear de Furhman más común fue el II y el tipo histológico el CR convencional, sin diferencias significativas al comparar los grupos definidos (Tablas 2 y 3). El análisis de la ploidía del ADN reveló mayor índice de aneuploidías en el grupo I de forma significativa $(\mathrm{p}=0,033)$, como se refleja en la Tabla 4 . Al comparar la ploidía del ADN según estadio y grupo de edad (Tabla 5), observamos que en general existe mayor porcentaje de aneuplodías en el grupo I para todos los estadios salvo el II. Sin embargo, estas diferencias sólo alcanzaron la significación estadística en los tumores en estadio I.

La comparación del tamaño tumoral estratificado según sus cuartiles para ambos grupos tampoco mostró diferencias significativas entre ambos $(p=0,670)$.

En el grupo II, 189 pacientes (96\%) se trataron mediante nefrectomía radical frente a los 100

Distribución de pacientes según grado y grupo de edad

$(\mathrm{x}=$ grado no establecido) .

\begin{tabular}{|c|c|c|c|c|c|}
\hline & $\begin{array}{c}\text { Grado X } \\
\text { n (\%) }\end{array}$ & Grado 1 & Grado 2 & Grado 3 & Grado 4 \\
\hline Edad $<65$ años & $3(1,52)$ & $64(32,4)$ & $67(34,01)$ & $42(21,31)$ & $21(10,65)$ \\
\hline Edad $>65$ años & $1(0,93)$ & $27(26,21)$ & $44(42,71)$ & $22(21,35)$ & $9(8,73)$ \\
\hline Valor $\mathrm{p}$ & & 0,82 & 0,30 & 0,13 & 0,46 \\
\hline \multicolumn{6}{|l|}{$\mathrm{p}=0,169$} \\
\hline \multicolumn{6}{|l|}{ Tabla 3} \\
\hline Dis & (2) & 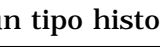 & o & & \\
\hline & \multicolumn{2}{|c|}{$\begin{array}{c}\text { CR convencional } \\
\text { n (\%) }\end{array}$} & $\begin{array}{c}\text { CR Papilar r } \\
\text { (\%) }\end{array}$ & \multicolumn{2}{|c|}{$\begin{array}{c}\text { CR Cromófobo n } \\
(\%)\end{array}$} \\
\hline Edad $<65$ años & \multicolumn{2}{|c|}{$128(65,3)$} & $42(21,31)$ & \multicolumn{2}{|c|}{$19(9,64)$} \\
\hline Edad > 65 años & \multicolumn{2}{|c|}{$71(68,93)$} & $21(20,38)$ & \multicolumn{2}{|c|}{$5(4,85)$} \\
\hline Valor p & \multicolumn{2}{|c|}{0,58} & 0,83 & \multicolumn{2}{|c|}{0,18} \\
\hline
\end{tabular}


Tabla 4

Distribución de pacientes según ploidía y grupo de edad

\begin{tabular}{lcc}
\hline & $\begin{array}{c}\text { Diploidia } \\
\mathbf{n}(\%)\end{array}$ & $\begin{array}{c}\text { Aneuploidia } \\
\mathbf{n}(\%)\end{array}$ \\
\hline Edad $<65$ años & $98(62,4)$ & $59(37,6)$ \\
Edad $>65$ años & $41(48,2)$ & $44(51,8)$ \\
Valor $p$ & 0,01 & 0,016 \\
\hline
\end{tabular}

pacientes (97\%) del grupo de más edad, sin diferencias significativas. Al resto se practicó nefrectomía parcial $(\mathrm{p}=0,83)$.

Durante el seguimiento a largo plazo, se evidenció progresión tumoral tumoral en 54 pacientes del grupo II (27\%) y en 27 (33,3\%) del grupo I $(p=0,061)$. Al analizar la progresión en función de la organoconfinidad del tumor al diagnóstico, observamos que estas diferencias sólo alcanzaban la significación cuando comparábamos tumores no órgano confinados, con mayor índice de progresión en los pacientes del grupo I ( $\mathrm{p}=0,003)$.

La supervivencia media cáncer específica en el grupo I fue de 40,42 meses y en el grupo II de 63 meses, siendo la diferencia estadísticamente significativa $(\mathrm{p}<0,001)$. La supervivencia actuarial global cáncer específica también fue diferente en función de la edad (Fig. 1), con mayor supervivencia de los pacientes del grupo II $(p=0,046)$. Esta diferencia en la supervivencia actuarial cáncer específica entre ambos grupos se evidenció tanto en el grupo de tumores órgano-confinados como en el de no órgano-confinados $(\mathrm{p}=0,026)$.

Ante estos resultados, analizamos si la edad era un factor pronóstico independiente de supervivencia y progresión. Sin embargo, el modelo de regresión de Cox no seleccionó la edad como factor pronóstico independiente de supervivencia. Sólo el TNM, el grado y la ploidía del ADN mostraron su valor pronóstico independiente de supervivencia en este modelo (Tabla 6).

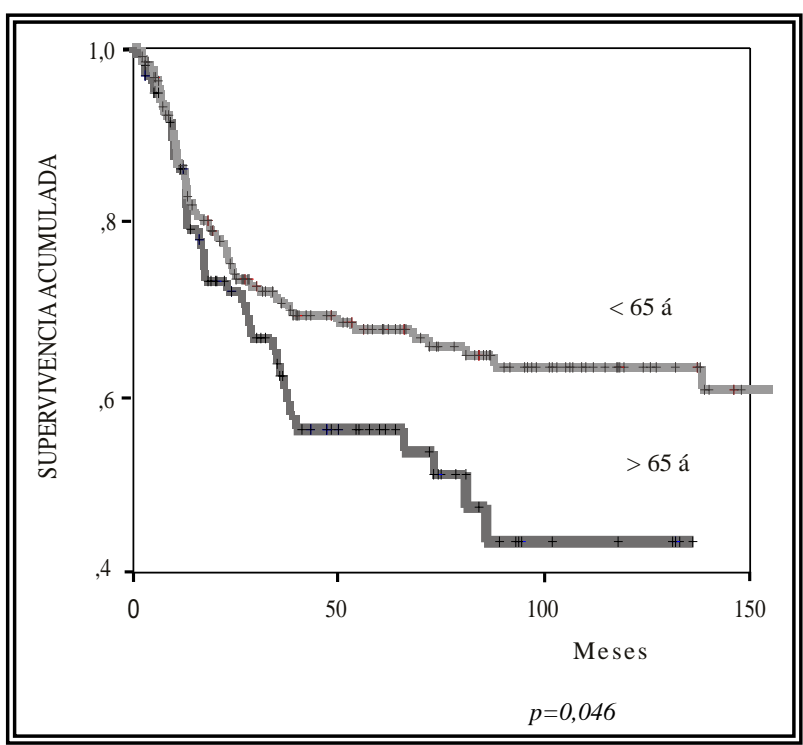

FIGURA 1. Supervivencia actuarial cáncer especifica se gún grupo de edad.

\section{DISCUSION}

Pocos autores han evaluado las características del CR en el grupo de edad geriátrica a pesar del progresivo incremento de los pacientes mayores de 65 años en nuestra sociedad ${ }^{3,12,13}$. De hecho, se pensaba que el riesgo demográfico del CR disminuiría después de los 65 años ${ }^{14}$, en contraste con el alto porcentaje de pacientes en edad geriátrica con CR observado en nuestra serie. En un estudio multicéntrico sobre 1.092 pacientes, Luciani et al. ${ }^{15}$ evidenciaron que la incidencia del $\mathrm{CR}$ se había duplicado en los pacientes mayores de 65 años. Este aumento de incidencia en edades avanzadas del CR podría ser debida al elevado número de exploraciones radiológicas que se realizan en la población añosa.

El estadio patológico es el factor pronóstico más importante del $\mathrm{CR}^{16}$. Rainwater et al. ${ }^{17}$ comparan 34 pacientes de 20 a 40 años con 41 pacientes mayores de 80 años y encuentran que los pacientes jóvenes presentaban tumores localizados con

Tabla 5

Distribución de pacientes según porcentaje de aneuploidías, estadio y grupo de edad

\begin{tabular}{lcccc}
\hline & $\begin{array}{c}\text { Estadio I } \\
\text { (\% aneuploidias) }\end{array}$ & $\begin{array}{c}\text { Estadio II } \\
\text { (\%aneuploidias) }\end{array}$ & $\begin{array}{c}\text { Estadio III } \\
\text { (\% aneuploidias) }\end{array}$ & $\begin{array}{c}\text { Estadio IV } \\
\text { (\% aneuploidias) }\end{array}$ \\
\hline Edad<65 años & $8(18,6)$ & $9(28,1)$ & $27(22,9)$ & $15(50)$ \\
Edad> 65 años & $10(50)$ & $4(22,2)$ & $22(52,9)$ & $8(66,7)$ \\
Valor p & 0,01 & 0,648 & 0,342 & 0,327 \\
\hline
\end{tabular}


Tabla 6

Valor pronóstico independiente de las variables estudiadas

\begin{tabular}{lcccccc}
\hline Variable & Coeficiente & E.E. & Wald & p-valor & Riesgo R. & I.C. 95\% \\
\hline $\begin{array}{l}\text { Diploide vs } \\
\text { aneuploide }\end{array}$ & 0,5077 & 0,2551 & 3,9600 & 0,0466 & 1,6615 & $1,00-2,73$ \\
$\begin{array}{l}\text { Organoconfinado vs } \\
\text { no organoconfinado }\end{array}$ & 1,3149 & 0,3181 & 17,0851 & 0,0000 & 3,7342 & $1,99-6,94$ \\
Tamaño & $-0,0363$ & 0,2445 & 0,0220 & 0,8821 & 0,9644 & $0,59-1,55$ \\
Grado I-II vs III-IV & 0,39322 & 0,2544 & 13,4232 & 0,0002 & 2,5402 & $1,54-4,18$ \\
$\leq 65$ vs > 65 años & 0,1993 & 0,03284 & 0,3681 & 0,5440 & 1,2205 & $0,64-2,32$ \\
\hline
\end{tabular}

mayor frecuencia y con mayor porcentaje de aneuploidías en el grupo de tumores órgano-confinados que los mayores de 80 años. Sin embargo, en nuestro estudio, el porcentaje de aneuploidías fue superior en el grupo de pacientes mayores de 65 años respecto a los menores para todos los estadios, excepto en el II, aunque con un diseño de estudio diferente, que los hace no comparables.

En un estudio reciente, Guillet et al. ${ }^{18}$ concluyó que el CR en pacientes de 18 a 40 años se presenta con más frecuencia como órgano confinado, de lo cual deriva una mayor supervivencia. Otros autores han encontrado una excelente supervivencia (del $85 \%$ al 93\%) en pacientes jóvenes con tumores localizados ${ }^{11}$. En todos estos trabajos se observa una tendencia hacia estadios localizados conforme disminuye la edad de los pacientes. A diferencia de éstos, en nuestra serie no encontramos diferencias significativas respecto al estadio patológico. Las posibles causas que expliquen estas diferencias son el punto de corte de edad seleccionado, que es arbitrario en la mayoría de los estudios, y la elevada mediana de edad de nuestra serie.

En nuestra línea de resultados Schiff et al. ${ }^{19}$ comparan 37 pacientes menores de 40 años con 486 mayores de 40 años, encontrando que el número de pacientes de más edad con tumores en estadio I es mayor que en el grupo de menor edad (63\% vs. $43 \%$ ), aunque la supervivencia global es casi el doble en los pacientes menores de 40 años con tumores órgano-confinados.

No encontramos diferencias entre los grupos de edad definidos respecto al tipo histológico ya que el subtipo más frecuente es el carcinoma convencional en ambos grupos, al igual que señalan el resto de autores ${ }^{11,17,20}$. Una excepción a esta tendencia son los resultados de Renshow ${ }^{10}$, que refiere que en 19 de los 24 pacientes (79\%) de su serie, la histología del tumor era del subtipo papilar. Sin embargo el $58 \%$ de sus pacientes tenían menos de 18 años, por lo que los resultados no son comparables a los nuestros. Al igual que en otras series ${ }^{3,21}$, la mayoría de los tumores estudiados eran de bajo grado (1 ó 2), con independencia de la edad.

Al igual que otros autores ${ }^{1}$ no encontramos diferencias en el tipo de cirugía (radical o parcial) realizada, aunque nuestro porcentaje de cirugias conservadoras es inferior. La explicación de este dato viene dada por la selección de los pacientes, ya que sólo incluimos aquellos con análisis de la ploidía del ADN tumoral, para lo cual se precisan tumores con un tamaño relativamente significativo, ya que en tumores de pequeño tamaño, susceptibles de tratamiento conservador, la sección y extracción de fragmentos puede distorsionar el informe anátomo-patológico definitivo.

$\mathrm{Al}$ analizar la progresión y su relación con la edad, encontramos diferentes conclusiones al revisar las series publicadas. Goetzl ${ }^{1}$, no encuentra diferencias en la tasa de recaídas al comparar pacientes menores y mayores de 40 años, siendo el tamaño tumoral la única variable que relaciona en el análisis multivariante con la progresión, pero sólo para el grupo de jóvenes. En nuestra serie hemos encontrado diferencias en la tasa de progresión, superior en el grupo en edad geriátrica con tumores no órgano confinados. Sánchez Ortiz $^{22}$ y Damhuis ${ }^{23}$ obtienen resultados similares, pero al contrario que nosotros, encuentran que la edad se comporta como una variable pronóstica independiente respecto a la progresión. Sin embargo no es posible precisar si este hecho sería consecuencia de la biología del propio tumor o de 
las características inmunológicas del anciano. En nuestra serie la edad carece de valor pronóstico de supervivencia.

Respecto a la supervivencia cáncer específica Boykin $^{11}$ obtiene una menor supervivencia en pacientes menores de 40 años en estadio avanzado. Pero su serie es muy corta (18 pacientes) y además no caracteriza al grupo de mayores de 40 años. La mayoría de estudios publicados reflejan peores supervivencias en pacientes añosos ${ }^{22}$. En concordancia con estos autores, hemos observado una menor supervivencia en los pacientes en edad geriátrica tanto en tumores localizados como no órgano confinados. Esta menor supervivencia del paciente geriátrico en nuestra serie, probablemente sea debida al mayor índice de aneuploidías detectado en los pacientes de más edad, ya que no existen diferencias entre ambos grupos en el resto de parámetros analizados.

\section{CONCLUSIONES}

La edad en el CR no tiene un valor pronóstico independiente de supervivencia. El CR renal en la edad geriátrica (mayores o iguales de 65 años), tiene unas características clínicas y patológicas similares al resto de pacientes en nuestra serie. Sin embargo, la supervivencia media y actuarial de este grupo de dad es menor, probablemente debido al mayor porcentaje de aneuploidias detectadas en este grupo.

\section{REFERENCIAS}

1. Goetzl MA, Desai M, Mansukhani M, Goluboff ET, Katz AE, Sawczuk IS, et al. Natural history and clinical outcome of sporadic renal cortical tumors. Urology 2004;63:41-45.

2. Lam J, Leppert J, Belldegrun A, Figlin R. Adjuvant teraphy of renal cell carcinoma: patient selection and therapeutic options. BJU 2005;96:483-488.

3. Griffiths DF, Verghese A, Golash A, Kynaston HG, Matthews PN, Hart AJ, et al. Contribution of grade, vascular invasion and age to outcome in clinically localized renal cell carcinoma. BJU Int 2002;90:26-31.

4. Ficarra V, Prayer-Galetti T, Novella G, Bratti E, Maffei N, Dal Bianco $\mathrm{M}$, et al. Incidental detection beyond pathological factors as prognostic predictor of renal cell carcinoma. Eur Urol 2003;43:663-669.

5. Mejean A, Hopirtean V, Bazin JP, Larousserie F, Benoit H, Chretien Y, et al. Prognostic factors for the survival of patients with papillary renal cell carcinoma: meaning of histological typing and multifocality. J Urol 2003;170:764-767.

6. Sanchez Zalabardo D, Arocena Garcia-Tapia J, Regojo Balboa JM, Fernandez Montero JM, Lopez Ferrandis J, Rosell Costa D, et al. [Prognosis factors in pT3 renal carcinoma]. Actas Urol Esp 2003;27:26-32.

7. Delahunt B. Histopathologic prognostic indicators for renal cell carcinoma. Sem Diag Pathol 1998;15:68-76.
8. Ruiz Cerdá JL, Hernández Marti M, Sempere Talens A, Martínez Jabaloyas J, Rodrigo Aliada M, Monros Lliso JL, et al. Estudio del contenido en $\mathrm{ADN}$ y de su asociación con el estadio, tamaño tumoral, arquitectura, tipo celular y grado nuclear en el adenocarcinoma renal. Actas Urol Esp 1997;21(1):6-14.

9. Lam J, Shvarts O, Leppert J, Figlin R, Belldegrun A. Renal cell carcinoma 2005: new frontiers in staging, prognostication, and targeted molecular therapy. J Urol 2005;173: 1853-1862.

10. Renshaw A, Granter S, Fletcher J, Kozakewich H, Corless C, Perez-Ataide A. Renal cell carcinomas in children and young adults: increased incidence of papillary architecture and unique subtypes. Am J Surg Pathol 1999;23:795-802.

11. Boykin WH, Bright KE, Zeidman EJ, Thompson IM. Renal tumors in young adults. Urology 1992;40:503-505.

12. Bretheau D, koulani A, Chevallier E. and the Oncology Committee of the Association Française d'Urologie: A French national epidemiologic survey on renal cell carcinoma. Cancer 1988;82:538-544.

13. Mermershtain W, Neulander E, Eidelberg I, Gusakova I, Kaneti J. Influence of age on the prognosis of patients with renal cell carcinoma. Onkologie 2002;25:548-550.

14. Paganini-Hill A, Ross R, Henderson B. Epidemiology of kidney cancer. In: Skinner D, editor. Urological cancer. New York: Grunne and Stratton, 1983:383-407.

15. Luciani L, Cestari R, Tallarigo C. Incidental renal cell carcinoma-age and stage characterization and clinical implications: study of 1092 patients (1982-1997). Urology 2001;57:206-207.

16. Doherty JG, Rufer A, Bartholomew P, Beaumont DM. The presentation, treatment and outcome of renal cell carcinoma in old age. Age Ageing 1999;28:359-562.

17. Rainwater LM, Zincke H, Farrow GM, Gonchoroff NJ. Renal cell carcinoma in young and old patients. Comparison of prognostic pathologic variables (cell type, tumor grade and stage and DNA ploidy pattern and their impact on disease outcome. Urology 1991;38:1-5.

18. Guillett M, Cheville J, Karnes N, Lohse C, Kwon E, Leibivich B, et al. Comparison of presentation and outcome for patients 18 to 40 and 60 to 70 years old with solid masses. J Urol 2005;173:1893-1896.

19. Schiff M, Herter G, Lytton B. Renal adenocarcinoma in young adults. Urology 1985;25:357-359.

20. Rodriguez A, Patard JJ, Lobel B. Renal cell carcinoma in adults less than 40 years of age: a particular cancer? Incidence, disease outcome and review of the literature. Ann Urol (Paris) 2003;37:155-159.

21. Eggener Smith ND, Nadler RB, Kontak J, Flanigan RC, Waters WB, Picken M, et al. Renal tumors in young adults. J Urol 2004;171:106-110.

22. Sanchez-Ortiz R, Rosser CJ, Madsen LT, Swanson DA, Wood CG. Young age is an independent prognostic factor for survival of sporadic renal cell carcinoma. J Urol 2004; 171:2160-2165.

23. Damhuis R, Blom JH. The influence of age on treatment choice and survival in 735 patients with. Br J Urol 1995; 75:143-147.

Dr. L. Gómez Pérez

E-mail: luisgope@hotmail.com

(Trabajo recibido el 14 de octubre 2005) 